\title{
Dynamic Vortex-Antivortex Interaction in a Single Cross-Tie Wall
}

\author{
K. Kuepper, ${ }^{1, *}$ M. Buess, ${ }^{2}$ J. Raabe, ${ }^{2}$ C. Quitmann, ${ }^{2}$ and J. Fassbender ${ }^{1}$ \\ ${ }^{1}$ Forschungszentrum Dresden-Rossendorf, Post Office Box 5101 19, D-01314 Dresden, Germany \\ ${ }^{2}$ Swiss Light Source, Paul Scherrer Institut, CH-5232 Villigen, Switzerland \\ (Received 1 March 2007; revised manuscript received 17 May 2007; published 18 October 2007)
}

\begin{abstract}
A fascinating property of micromagnetism comes from the possibility to control the domain and vortex configuration through the sample shape and size. For instance, in a rectangular platelet a configuration containing a stable combination of vortices and an antivortex can be created. Such a single cross-tie wall can be understood as being a coupled micromagnetic system with three static solitons. Here we report on its magnetization dynamics including the vortex-antivortex interactions. The spectrum of eigenmodes is investigated as well as the effect of different vortex core orientations. We show that the vortex dynamics can be used to identify the core configuration, which is not directly accessible to x-ray microscopy because of its limited spatial resolution.
\end{abstract}

DOI: 10.1103/PhysRevLett.99.167202

PACS numbers: 75.40.Gb, 75.60.Ch, 75.75.+a

Two-dimensional topological solitons are fascinating for researchers in many fields. These solitons determine the properties of very different systems such as atoms in superfluids and Bose-Einstein condensates [1,2] and Cooper pairs in superconductors. In thin ferromagnetic films they can be present as vortices and antivortices [3-7]. Because vortex and antivortex are the corresponding antiparticles they can annihilate under emission of energy [8]. However, in special geometries a stable combination of vortices and antivortices can be obtained. A cross-tie wall is an example of an infinite chain of vortices and antivortices.

Here we study a ferromagnetic rectangle, containing two vortices and a single antivortex, thus forming the unit cell of a cross-tie wall. The solitons are coupled through the domain walls and domains. This special geometry provides insight into the vortex configuration and into coupling effects which turn out to be very relevant for the dynamics of these solitons.

Up to now, significant effort has been invested in understanding the dynamics of "simple" magnetic vortex structures, such as thin permalloy squares and disks. Timeresolved imaging $[9,10]$ allows us to investigate their excitations and switching in the time-domain [11-14]. Vortex-antivortex interaction has also attracted attention in semicontinuous films because of potential applications for spin wave radiation devices $[8,15]$, and magnetic pinning arrays for superconducting films [16].

Neudert et al. [17] have investigated the generation of cross-tie walls in large permalloy platelets following the excitation by field pulses using Kerr microscopy. In this Letter we investigate the excitations of a single cross-tie wall by combining micromagnetic simulations based on the LLG equation [18] and magnetic imaging by means of time-resolved photo emission electron microscopy (PEEM). We study the response to weak magnetic in-plane field pulses, concentrating on the dynamics of the vortices and the antivortex and their mutual interaction.
Permalloy $\left(\mathrm{Ni}_{81} \mathrm{Fe}_{19}\right)$ samples of $50 \mu \mathrm{m}$ length, $6 \mu \mathrm{m}$ width, and $20 \mathrm{~nm}$ thickness were prepared on a $10 \mu \mathrm{m}$ wide coplanar waveguide. A rectangular element of $10 \times$ $6 \mu \mathrm{m}^{2}$ was patterned by focused ion beam (FIB) sputtering. The time and spatially resolved magnetization was measured using PEEM. Employing $x$-ray magnetic circular dichroism at the $\mathrm{Fe} L_{3}$ edge the image intensity is $I \propto$ $M_{y}(\vec{r}) \cdot \vec{P}$. We use a gray scale representation with white representing the parallel and black the antiparallel orientation of the magnetization and the polarization. The rectangle is excited every 16 ns using magnetic field pulses synchronized to the $\mathrm{x}$-ray pulses emitted by the synchrotron. The time dependence is measured by varying the time delay $\Delta t[12]$. The field pulse $\vec{H}_{p}$ is along the $y$ direction and has a magnitude of $\approx 20$ Oe and a temporal width of 500 ps with a rise time of about 150 ps.

First we discuss the equilibrium magnetization of a ferromagnetic platelet containing a single cross-tie wall. Figure 1(a) shows the simulated in-plane component $M_{y}(\vec{r})$ of the magnetization. The magnetization configuration can be thought of as consisting of two squares, each having a clockwise flux-closure pattern and containing a vortex core in its center. The two flux-closure patterns are separated by

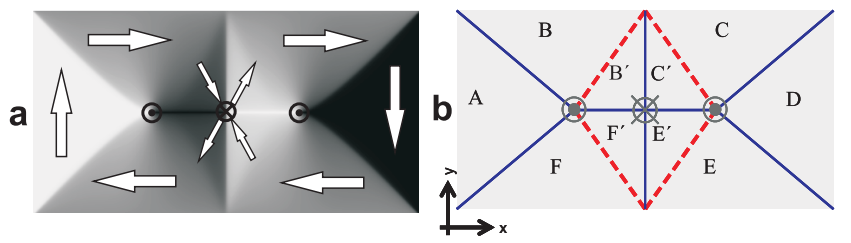

FIG. 1 (color online). (a) Micromagnetic simulation showing $M_{y}(\vec{r})$ for a rectangular platelet $(5 \mu \mathrm{m} \times 3 \mu \mathrm{m} \times 20 \mathrm{~nm})$ containing a single cross-tie wall. (b) Sketch depicting the $90^{\circ}$ Néel walls (solid lines, blue) and the $45^{\circ}$ pseudo Néel walls (dashed lines, red). The vortex cores have an out of plane magnetization with positive $(u$ or $\odot$ ) or negative $(d$ or $\otimes) z$ direction. Here we find $[u d u]$. 
a Néel wall of $\sim 90^{\circ}$ running along the $y$ axis and containing the antivortex at the center. A second Néel wall connecting the two vortex cores runs along the $x$ axis [see Fig. 1(b)]. The cross-tie structure consists of these $90^{\circ}$ Néel walls and four $45^{\circ}$ pseudo Néel walls (dashed lines). The latter are pseudo domain walls, because along them the magnetization rotates continuously (e.g., between domains $B$ and $B^{\prime}$ ). In addition there are four $90^{\circ}$ Néel walls (solid lines) running from the platelet edges to the vortex cores. To reduce the exchange energy that would be associated with spins on neighboring atoms pointing antiparallel, the magnetization of the vortex and antivortex rotates out of plane in a narrow region called the core. This core can point either in positive $(u$ or $\odot)$ or in negative $(d$ or $\otimes) z$ direction resulting in a total of $2^{3}$ possible configurations.

PEEM images of the magnetization $M_{y}(\vec{r}, \Delta t)$ of the permalloy rectangle $(10 \mu \mathrm{m} \times 6 \mu \mathrm{m} \times 20 \mathrm{~nm})$ are shown in Fig. 2 (for a movie of the full series see Ref. [19]). The top row displays the experimental data, the second row the corresponding micromagnetic simulations. The two subsequent rows show difference images $M_{y}(\vec{r}, \Delta t)-M_{y}(\vec{r}, 0)$ for experiment and simulation, respectively. These difference images visualize the changes relative to the equilibrium state. In our geometry, the short edge of the rectangle is along the $y$ direction and parallel to both the x-ray polarization $\vec{P}$ and the magnetic field pulse $\vec{H}_{p}$.

The first image shows the equilibrium state of the magnetization $(\Delta t=0)$. The domains $A-F$ are similar to domains in conventional flux-closure patterns. Domain $A$, which is parallel to $\hat{y}$, is white and domain $D$, which is antiparallel, is black. Domains $B, C, E$, and $F$ are gray since their magnetization is perpendicular to $\hat{y}$. The domains $B^{\prime}, F^{\prime}, C^{\prime}$, and $E^{\prime}$ in the cross-tie are at $\pm 45^{\circ}$ to $\hat{y}$ resulting in darker and lighter gray values, respectively.

Next we discuss the temporal evolution of the magnetization. At a delay of $\Delta t=300 \mathrm{ps}$ all domains having a finite $x$ component of the magnetization in the equilibrium state have become brighter. The reason is the torque $(\vec{M} \times$ $\vec{H}_{p}$ ) exerted by the field pulse causing an excursion in the $z$ direction followed by a precession. This rotates their magnetization into the $y$ direction leading to a higher intensity. Only domains $A$ and $D$ do not show such an increased intensity because they are parallel and antiparallel to the field pulse, respectively.

In addition the $90^{\circ}$ Néel walls originating from the fluxclosure pattern and the cross-tie wall pointing along $\hat{y}$ bulge to the right. In the difference images these effects show up as bright areas and lines, respectively. At $\Delta t=$ $450 \mathrm{ps}$ the coherent precession in the domains has continued. The magnetization is almost perpendicular to $\hat{y}$, resulting in a gray difference intensity. The bulged domain walls are now even more intense than at $\Delta t=300 \mathrm{ps}$ and are visible in experiment and simulation. At $\Delta t=600 \mathrm{ps}$ the ongoing precession leads to a brighter appearance of domains $B, C, E$, and $F$ again. For this delay time we observe a maximum in the curvature of both, the cross-tie

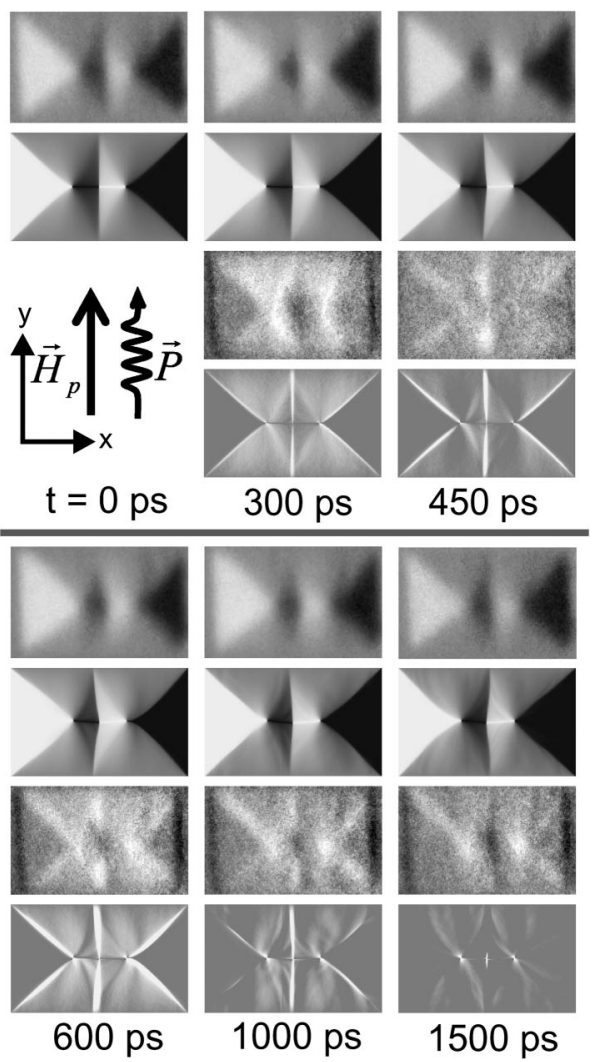

FIG. 2. Magnetization $M_{y}(\vec{r}, \Delta t)$ at characteristic delay times. Experimental results (top row) and difference images (third row) are compared to the corresponding simulation (second and fourth row).

wall along $\hat{y}$ and the $90^{\circ}$ Néel walls along the black $(D)$ and white $(A)$ domains.

Turning to longer delays, the vortex dynamics becomes prominent. The difference images show that the Landau like domains $A-F$ have almost completely relaxed back to the equilibrium state. The domain walls, the two vortices, and the antivortex, on the other hand, are still displaced, resulting in a finite intensity for the difference images. For $1500 \mathrm{ps}$, the main visible features are the two vortices and the antivortex, indicating their slow relaxation into the equilibrium state. For all delays we find reasonable agreement between simulation and experiment [20].

To gain further insight into the dynamics we perform a Fourier transformation [21,22] of the experimental data, using a Hamming cutoff window. Large Fourier amplitudes indicate eigenfrequencies of the system. Figure 3 shows the spatial distribution of the Fourier amplitude and phase for the observed eigenfrequencies. For the higher frequencies $(2.2$ and $2.4 \mathrm{GHz})$ the intensity dominates in the domains $B, C, E$, and $F$, all of which have their equilibrium magnetization perpendicular to $\vec{H}_{p}$. The frequencies and the observed phase shift of $\pi$ (corresponding to a change from red to blue in Fig. 3) are in good agreement with the findings in Landau flux-closure structures [12]. 

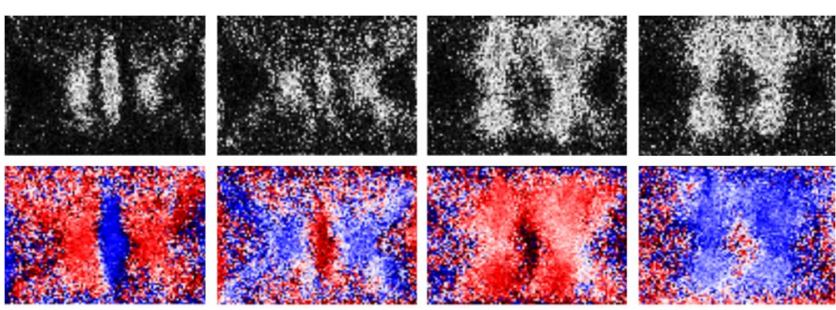

$0.9 \mathrm{GHz}$

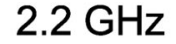

\section{$2.4 \mathrm{GHz}$}

FIG. 3 (color online). Spatial distribution of the Fourier components of the magnetization $M_{y}(\vec{r})$ at characteristic frequencies showing amplitude (top) and phase (bottom: dark gray, blue online $=0$, light gray, red online $=\pi$ ).

The wall modes occur at lower frequencies. For $1.1 \mathrm{GHz}$ we find large Fourier amplitudes around the two vortices, the antivortex positions, and the corresponding domain walls. In addition they seem to radiate out along the $90^{\circ}$ Néel walls, indicating the coupling of the vortices to these domain walls. At $0.9 \mathrm{GHz}$, corresponding to the longest time scale visible in our experiment, the dominant feature is the cross-tie wall along $\hat{y}$ indicating that it is the last subcomponent of the pattern responding to the exciting field pulse. This wall mode at $0.9 \mathrm{GHz}$ is phase shifted by $\pi$ with respect to the mode at $1.1 \mathrm{GHz}$. For both low frequency modes it is evident that the movement of the core positions is coupled to the adjacent domain walls, which mediates the mutual interaction of different cores by the exchange interaction within the wall.

Having studied the response of the domains and the domain walls we now focus on the vortex core dynamics and the effect of different core configurations. Our rectangle with the cross-tie wall containing two vortices and a single antivortex can have $2^{3}=8$ different configurations. There are two configurations where both vortex cores have the same orientation, but are opposite to the antivortex (I): $[u d u],[d u d]$. There are four configurations where both vortex cores are antiparallel (II): $[u u d],[u d d],[d u u]$, $[d d u]$. And last there are two configurations where all three cores are parallel (III): [uuu], [ddd].

Comparing the three configurations their total energies are very similar $\left(E_{u d u}=1.1254 \times 10^{-9} \mathrm{erg}, \quad E_{d u u}=\right.$ $\left.1.1392 \times 10^{-9} \mathrm{erg}, E_{\text {иии }}=1.1392 \times 10^{-9} \mathrm{erg}\right)$. Their total energies differ only about $1 \%$, and thus the present configuration is a priori not clear; however, the contribution of the exchange and demagnetizing energy differ substantially, indicating a change in the coupling between the vortex cores [19]. The core orientation is relevant since it determines the sense of rotation for the gyrotropic motion. Note that for the same orientation vortex and antivortex have an opposite sense of rotation [8]. A first example of a vortex-vortex interaction has been demonstrated by Buchanan et al. [23] for the case of a two-vortex system. Here we study the interaction in a system containing both vortex and antivortex cores.

From the simulated time series we extract the vortex and antivortex core positions. Figure 4(a) shows the displacements for the three different configurations (I, II and III) [18]. The vortex and antivortex motions show a very different behavior depending on the configuration. For configuration (I) [Fig. 4(a), top row] all three cores have the same sense of rotation [counterclockwise (ccw)], and the amplitudes and frequencies are similar. However, the antivortex exhibits a $180^{\circ}$ phase shift. This opposite motion of vortex and antivortex cores might also be called an optical mode. For configuration (II) the vortex cores point along opposite directions. This leads to different interaction with the antivortex. Compared to configuration (I), in particular, the antivortex and the right vortex core exhibit a significantly reduced gyration. For configuration (III) an
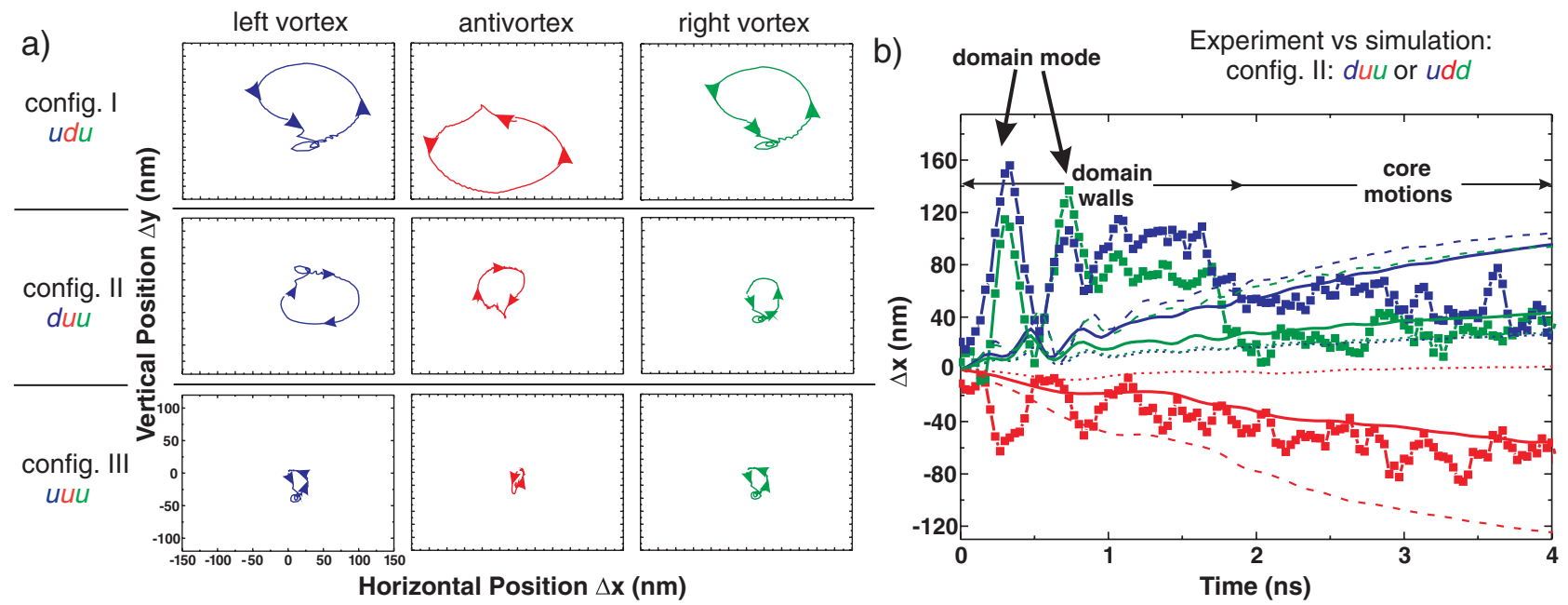

FIG. 4 (color online). (a) Trajectories of the two vortex (left and right, blue and green online) and the antivortex (center, red online) core displacements as extracted from micromagnetic simulations [18] ( $\Delta t=0-16 \mathrm{ns)}$ (see text). The arrows indicate the sense of rotation for the core motion. (b) Horizontal core displacement from experiment ( $\mathbf{\square}$ ) and the three possible configurations [configuration (I) (dashed line), configuration (II) (solid line), and configuration (III) (dotted line)]. 
even more complex behavior is observed. The amplitudes of all three cores are even more reduced. The two vortex cores gyrate as expected (ccw) and again have comparable amplitudes. The antivortex core gyration is, however, opposite from what was expected and its amplitude is reduced significantly. The reason is the interaction with the adjacent vortex cores, quenching the free gyration of the antivortex. The remaining five configurations can be deduced by means of symmetry considerations, provided the symmetry breaking caused by the direction of the pulse field $H_{p}$ is neglected.

From the above results it is evident that the vortexantivortex interaction, mediated by the exchange interaction of the corresponding domain walls, is very important for the magnetization dynamics of a single cross-tie wall. All other systems comprising multiple vortex and/or antivortex structures are likely to exhibit an even more complex dynamic behavior which can only be explained with a detailed understanding of the individual constituents.

To finally determine which of the $2^{3}$ possible core configurations is present in our experiment we make use of the strong configuration dependence of the dynamics presented in Fig. 4(a). The horizontal vortex displacements determined from experiment and simulations are compared in Fig. 4(b).

For configuration (II) we observe an agreement in the horizontal displacement direction for all three moving cores and in addition comparable amplitudes for $\Delta t \gtrsim$ $2 \mathrm{~ns}$. For $\Delta t \lesssim 2 \mathrm{~ns}$ the movements of the domain and the domain walls are strongly overlapping the vortex and antivortex movements (see Fig. 2) leading to a large uncertainty in the experimental determination of the core position. The horizontal displacement of the antivortex and the right vortex is very well reproduced, whereas the left vortex shows a somewhat larger displacement in the simulation; however, this deviation is comparable to the precision of the core determination of about $30 \mathrm{~nm}$.

An analysis of the vertical displacement is more difficult because of the reduced amplitudes and is not shown. We can therefore not distinguish between a $[d u u]$ and a $[u d d]$ configuration consistent with the horizontal displacement observed for configuration (II). However, we can conclude that one vortex is antiparallel to the antivortex and the other vortex core in the present experiment.

In summary, we report results of vortex-antivortex dynamics. These include the dynamics of antivortices and the indirect coupling between vortex cores and the antivortex, which is mediated by the exchange interaction of the adjacent domain walls. This coupling is significant and introduces unexpected effects, such as the quenching of gyrotropic motion for the antivortex in certain core configurations. Another consequence is the absence of simple eigenmodes describing the vortex gyration. By using simulations the strong influence of the vortex coupling on the core dynamics can in turn be used to determine the core configuration, a parameter otherwise inaccessible to direct observation.

Part of this work has been performed at the Swiss Light Source, Paul Scherrer Institut, Villigen, Switzerland. We are grateful to L. Bischoff for performing FIB. We thank S. Wintz, B. Liedke, M.R. Scheinfein, and C.H. Back for support and helpful discussions. We are indebted to D. Weiss (University of Regensburg) for making the cleanroom available.

\section{*k.kuepper@fzd.de}

[1] D. R. Tilley and J. Tilley, Superfluidity and Superconductivity (IOP Publishing Ltd., Bristol, 1990), 3rd ed..

[2] M. R. Matthews et al., Phys. Rev. Lett. 83, 2498 (1999).

[3] G. Blatter et al., Rev. Mod. Phys. 66, 1125 (1994).

[4] C. Kittel, Rev. Mod. Phys. 21, 541 (1949).

[5] A. Hubert and R. Schäfer, Magnetic Domains (Springer, Berlin, 1998).

[6] J. Raabe et al., J. Appl. Phys. 88, 4437 (2000).

[7] A. Wachowiak et al., Science 298, 577 (2002).

[8] R. Hertel and C. M. Schneider, Phys. Rev. Lett. 97, 177202 (2006).

[9] Y. Acremann et al., Science 290, 492 (2000).

[10] T. Gerrits et al., Nature (London) 418, 509 (2002).

[11] S.-B. Choe et al., Science 304, 420 (2004).

[12] J. Raabe et al., Phys. Rev. Lett. 94, 217204 (2005).

[13] K. Kuepper et al., Appl. Phys. Lett. 90, 062506 (2007).

[14] B. van Waeyenberge et al., Nature (London) 444, 461 (2006).

[15] S.-K. Kim et al., Appl. Phys. Lett. 86, 052504 (2005).

[16] M. V. Milošević and F. M. Peeters, Phys. Rev. Lett. 93, 267006 (2004).

[17] A. Neudert et al., J. Appl. Phys. 99, 08F302 (2006).

[18] http://llgmicro.home.mindspring.com We use standard permalloy material parameters: exchange constant $A=$ $8 \times 10^{-12} \mathrm{~J} / \mathrm{m}$, saturation magnetization $M_{s}=$ $860.000 \mathrm{~A} / \mathrm{m}$, magnetic damping constant $\alpha=0.01$, uniaxial anisotropy $K_{u}=0$. To take into account a possible resonant excitation we simulate a sequence of five full excitation cycles (every $16 \mathrm{~ns}$ ) for each configuration. We observe no significant change in the frequencies or the trajectories of the vortex cores, only the amplitudes of the trajectories increase. For the analysis we use the averaged results of the third-fifth excitation cycles.

[19] See EPAPS Document No. E-PRLTAO-99-053742 for a table displaying the energies of the three configuations and a movie of the data shown in Fig. 2. For more information on EPAPS, see http://www.aip.org/pubservs/epaps.html.

[20] Simulations were done for a rectangle of $(5 \mu \mathrm{m} \times$ $3 \mu \mathrm{m} \times 20 \mathrm{~nm}$ ) and a cell size of $5 \times 5 \times 20 \mathrm{~nm}^{3}$ to limit the neccessary memory and and computing times. The experiments are done on larger rectangles of $(10 \mu \mathrm{m} \times 6 \mu \mathrm{m} \times 20 \mathrm{~nm})$. We find good agreement between simulation and experiment justifying reduced size for the simulation.

[21] J. P. Park et al., Phys. Rev. B 67, 020403 (2003).

[22] M. Buess et al., Phys. Rev. Lett. 93, 077207 (2004).

[23] K. S. Buchanan et al., Nature Phys. 1, 172 (2005). 